\title{
Behavior of Sulfur Oxides in Nonferrous Metal Smelters and Implications on Future Control and Emission Estimation
}

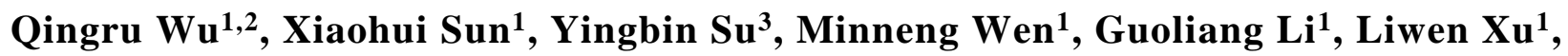
Zhijian Li ${ }^{1}$, Yujia Ren ${ }^{1}$, Jing Zou ${ }^{1}$, Haotian Zheng ${ }^{1}$, Yi Tang ${ }^{1}$, Lei Duan ${ }^{1,2}$, Shuxiao Wang ${ }^{1,2, *}$, Qin Zhang ${ }^{3}$

1 State Key Joint Laboratory of Environmental Simulation and Pollution Control, School of Environment, Tsinghua University, Beijing 100084, China

2 State Environmental Protection Key Laboratory of Sources and Control of Air Pollution Complex, Beijing 100084, China

3 School of Metallurgy, Northeastern University, Shenyang, 110819 Liaoning, China

*Corresponding authors.

Shuxiao Wang

Tel.: +86 1062771466; fax: +861062773597.

E-mail address: shxwang@tsinghua.edu.cn

Qin Zhang

Tel.: +86 18602420020; fax: +86 2423906316 .

E-mail address: zhangq@smm.neu.edu.cn

8 pages (including cover page)

4 tables (S1, S2, S3, S4)

2 figure (S1, S2) 


\section{S1. Smelter flow chart, sampling sites, and operation parameters}

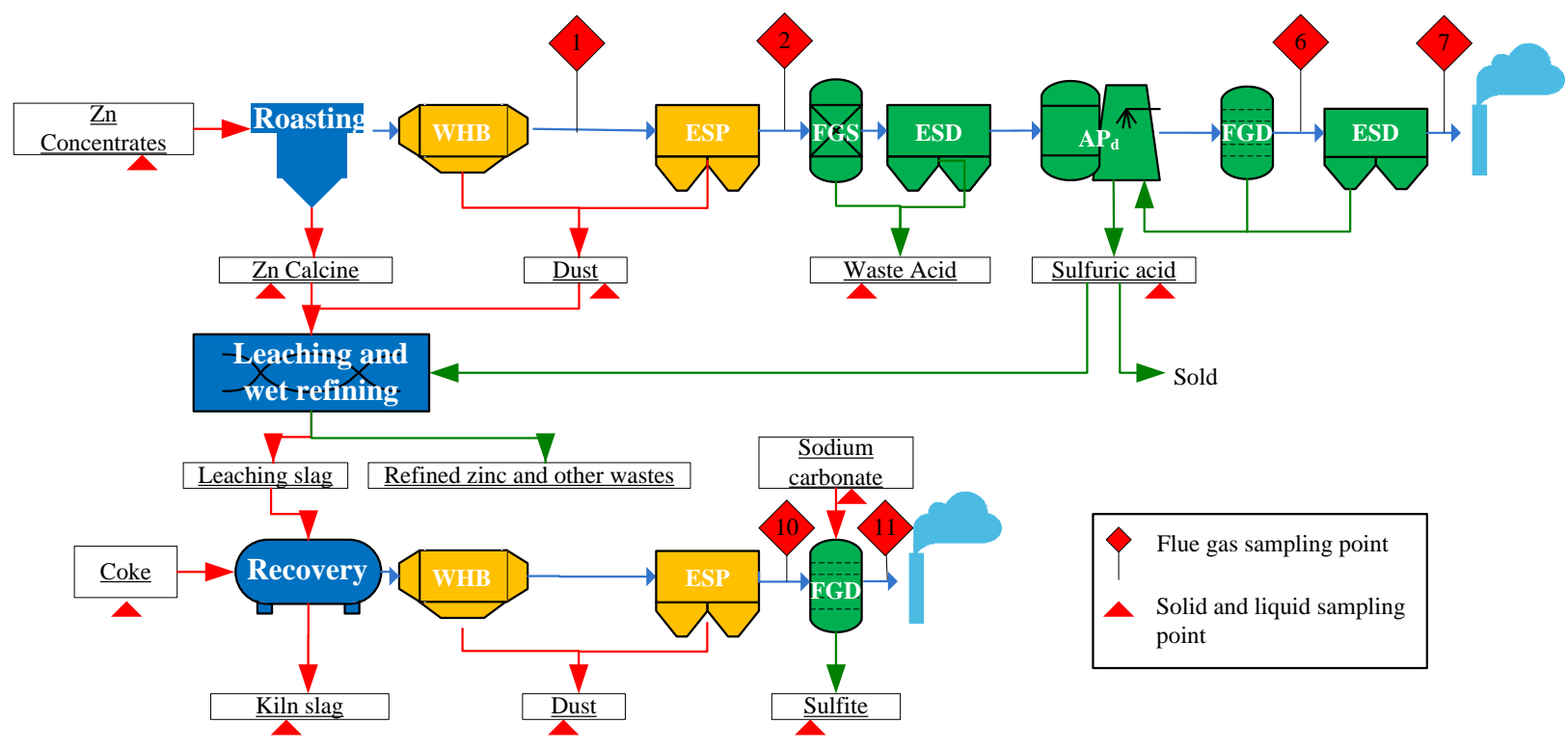

(a)

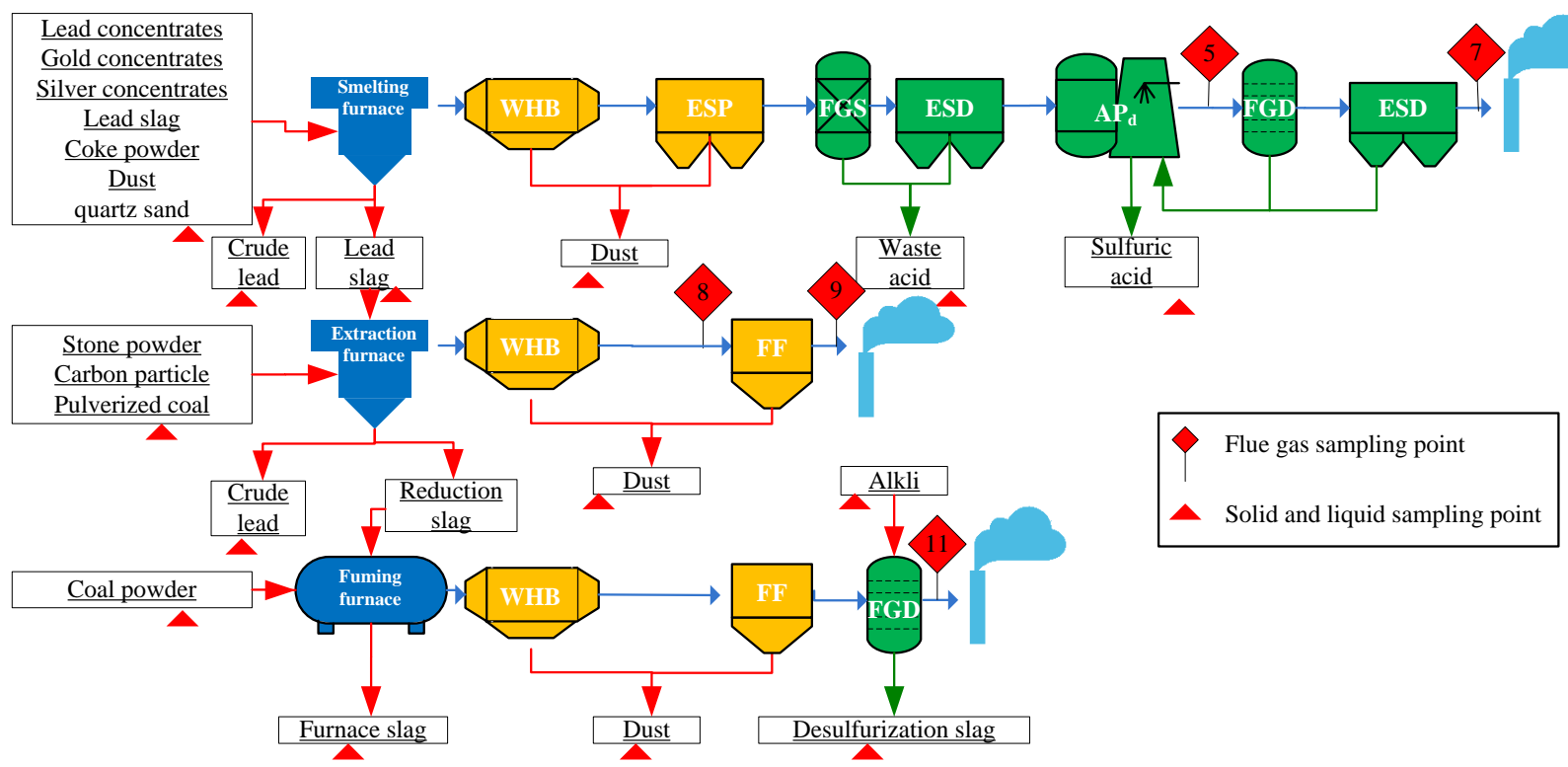

(b) 


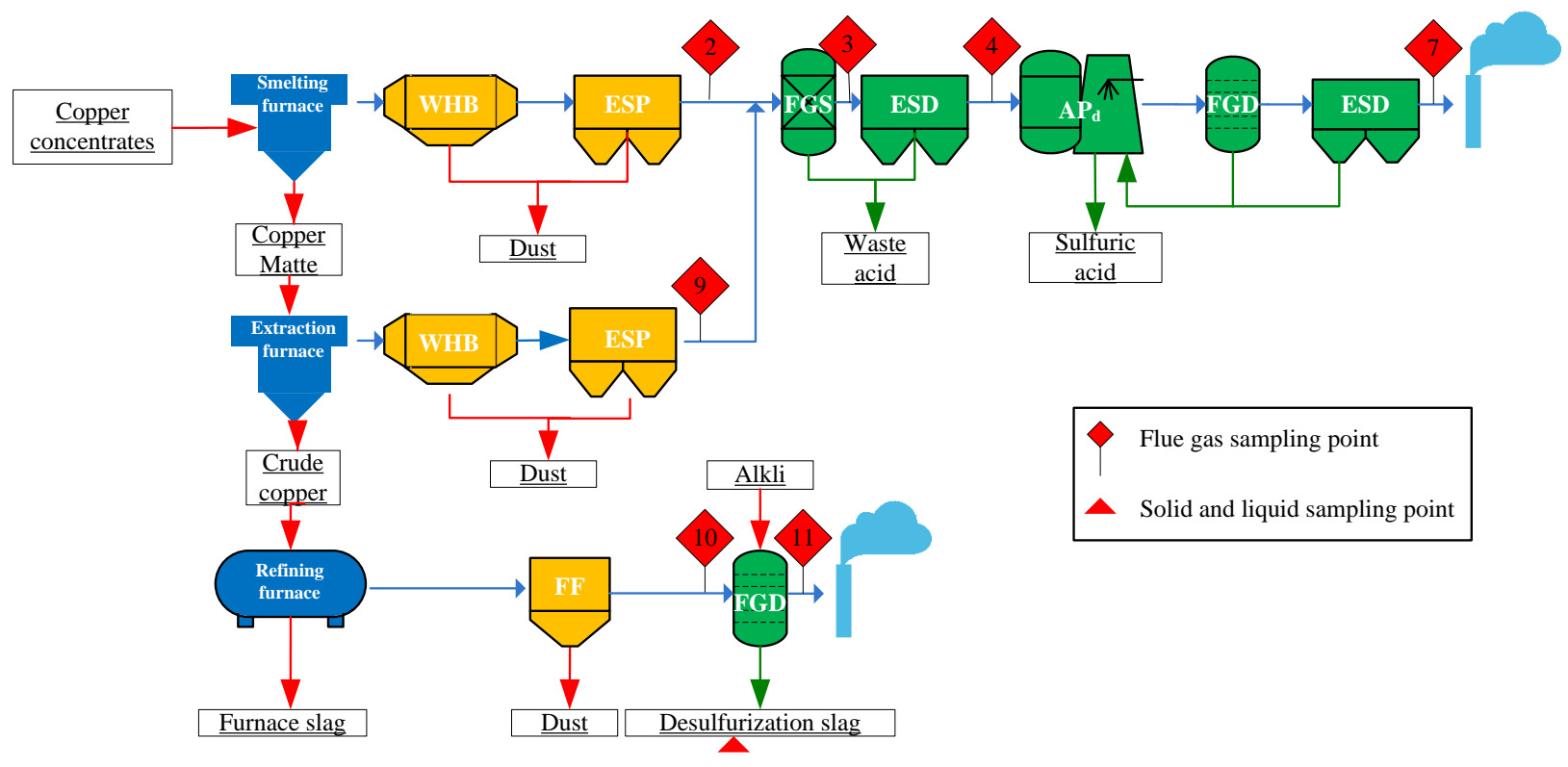

(c)

Figure S1. Sampling sites and samples in smelter A, B, and C. 
Table S1 Description of the studied smelters

\begin{tabular}{|c|c|c|c|c|c|c|}
\hline Smelter & Product & Technology & $\begin{array}{l}\text { Capacity } \\
(\mathrm{kt})\end{array}$ & $\begin{array}{l}\text { Furnace } \\
\text { temperature } \\
\left({ }^{\circ} \mathrm{C}\right),\end{array}$ & $\begin{array}{l}\text { Dust } \\
\text { production } \\
\text { rate }(\%)\end{array}$ & $\begin{array}{l}\mathrm{O}_{2} \\
\text { content } \\
(\%)\end{array}$ \\
\hline Smelter A & Zinc & Electrolytic process & 280 & 940 & 30 & 6 \\
\hline Smelter B & Lead & $\begin{array}{l}\text { Rich-oxygen pool } \\
\text { smelting }\end{array}$ & 400 & $1050-1150$ & 15 & 4 \\
\hline Smelter C & Copper & Flash furnace smelting & 100 & $1200-1300$ & 6 & 2.5 \\
\hline
\end{tabular}


Table S2 Site temperature and dew point

\begin{tabular}{|c|c|c|c|c|c|c|c|}
\hline \multirow{2}{*}{\multicolumn{2}{|c|}{$\begin{array}{l}\text { Site } \\
\text { description }\end{array}$}} & \multicolumn{3}{|l|}{ Smelter A } & \multicolumn{3}{|l|}{ Smelter C } \\
\hline & & \multirow{2}{*}{$\begin{array}{l}\text { Temperature } \\
\left({ }^{\circ} \mathrm{C}\right) \\
318\end{array}$} & \multirow{2}{*}{$\begin{array}{l}\text { Water vapor } \\
\left(\mathrm{mg} / \mathrm{m}^{3}\right)\end{array}$} & \multirow{2}{*}{$\begin{array}{l}\begin{array}{l}\text { Dew point } \\
\left({ }^{\circ} \mathrm{C}\right)\end{array} \\
145\end{array}$} & \multirow{2}{*}{$\begin{array}{l}\text { Temperature } \\
\left({ }^{\circ} \mathrm{C}\right) \\
\mathrm{NT}\end{array}$} & \multirow{2}{*}{$\begin{array}{l}\text { Water vapor } \\
\left(\mathrm{mg} / \mathrm{m}^{3}\right)\end{array}$} & \multirow{2}{*}{$\begin{array}{l}\text { Dew point } \\
\left({ }^{\circ} \mathrm{C}\right)\end{array}$} \\
\hline NO.1 & After WHB 1 & & & & & & \\
\hline NO.2 & After ESP 1 & 303 & 1135 & 136 & 310 & 921 & 110 \\
\hline NO.3 & After FGS 1 & NT & NT & NT & 43 & 345 & 89 \\
\hline NO.4 & After ESD 1 & NT & NT & NT & 34 & 32 & 57 \\
\hline NO.5 & After DCDA & 25 & 0.5 & 32 & 25 & 0.4 & 22 \\
\hline NO.6 & After FGD 1 & 26 & 97 & 61 & NT & NT & NT \\
\hline NO.7 & After ESD 2 & 25 & 86 & 48 & 24 & 5 & 19 \\
\hline NO.9 & After ESP 2 & NT & NT & NT & 293 & 597 & 121 \\
\hline NO.10 & After ESP/FF & 308 & 413 & 102 & 26 & 8 & 59 \\
\hline NO.11 & After FGD 2 & 62 & 430 & 66 & 27 & 35 & 33 \\
\hline
\end{tabular}

Note: NT represents that the concentrations were not tested due to no suitable sampling condition; WHB - waste heat boilers; ESP - electrostatic precipitator; FGS - flue gas scrubber; ESD - electrostatic demister; DCDA - double contact and double absorption tower; FGD - flue gas desulfurization tower. 


\section{S2. Parameters for the establishment of SOX emission inventory}

Table S3 The application proportion and unabated emission factors for different technologies

\begin{tabular}{|c|c|c|c|c|c|c|c|c|}
\hline \multirow{2}{*}{ Processes } & \multicolumn{7}{|c|}{ Application proportion, $\%$} & \multirow{2}{*}{$\begin{array}{l}\text { UEF for SOX } \\
\text { (kg/t product })\end{array}$} \\
\hline & Processes & 1978 & 1990 & 2000 & 2005 & 2010 & 2014 & \\
\hline \multirow{5}{*}{$\begin{array}{l}\text { Zinc } \\
\text { smelting }\end{array}$} & AZSP & 20.0 & 1.7 & 1.6 & 1.6 & 1.2 & 0.5 & 1515 \\
\hline & $\mathrm{EP}$ & 48.4 & 55.2 & 67.6 & 71.0 & 81.3 & 94.4 & 1264 \\
\hline & ISP & 14.0 & 17.8 & 10.1 & 12.0 & 7.3 & 2.2 & 1176 \\
\hline & RZSP & 17.6 & 24.9 & 16.8 & 11.2 & 8.0 & 1.8 & 1515 \\
\hline & EZF & 0.0 & 0.3 & 3.9 & 4.2 & 2.2 & 1.0 & 1208 \\
\hline \multirow{4}{*}{$\begin{array}{l}\text { Lead } \\
\text { smelting }\end{array}$} & SPP & 89.1 & 81.6 & 68.2 & 66.7 & 32.5 & 14.2 & 582 \\
\hline & SMP & 10.9 & 12.9 & 24.5 & 29.0 & 21.7 & 20.8 & 582 \\
\hline & ISP & 0.0 & 5.5 & 7.3 & 4.3 & 4.7 & 4.2 & 573 \\
\hline & RPSP & 0.0 & 0.0 & 0.0 & 0.0 & 41.1 & 60.8 & 545 \\
\hline \multirow{5}{*}{$\begin{array}{l}\text { Copper } \\
\text { smelting }\end{array}$} & $\mathrm{EF} / \mathrm{RF}$ & 67.6 & 45.4 & 24.1 & 20.9 & 3.4 & 0.0 & 1994 \\
\hline & FFSP & 0.0 & 21.3 & 33.9 & 25.6 & 34.2 & 38.4 & 2151 \\
\hline & IFSP & 28.1 & 28.1 & 36.2 & 21.9 & 9.8 & 2.0 & 1994 \\
\hline & RLEP & 0.0 & 0.0 & 0.0 & 0.1 & 0.2 & 1.0 & 1994 \\
\hline & RPSP & 10.0 & 10.9 & 5.8 & 31.5 & 52.4 & 58.6 & 1912 \\
\hline
\end{tabular}

Note: UEF - unabated emission factor; AZSP - artisanal zinc smelting process; EP electrolytic process; ISP - imperial smelting process; RPSP - rich-oxygen pool smelting process; RZSP - retort zinc smelting process; EZF - electric zinc furnace; SPP - sinter pan or pot process; SMP - sinter machine process; EF/RF - electric furnace smelting process and the revelatory furnace smelting process; IFSP - imperial furnace smelting process; RLEP roasting-leaching-electrolyzing process; ISP-imperial sinter process. 
Table S4 Removal efficiencies and speciation profiles for different APCD combinations

\begin{tabular}{|c|c|c|c|c|c|c|c|}
\hline \multirow{3}{*}{ APCD combinations } & \multirow{3}{*}{$\begin{array}{l}\text { Removal } \\
\text { efficiency }(\%)\end{array}$} & \multicolumn{6}{|c|}{ Speciation profiles $(\%)$} \\
\hline & & \multicolumn{2}{|c|}{ Zinc smelting } & \multicolumn{2}{|c|}{ Lead smelting } & \multicolumn{2}{|c|}{ Copper smelting } \\
\hline & & $\mathrm{SO}_{3}$ & $\mathrm{SO}_{2}$ & $\mathrm{SO}_{3}$ & $\mathrm{SO}_{2}$ & $\mathrm{SO}_{3}$ & $\mathrm{SO}_{2}$ \\
\hline \multicolumn{8}{|l|}{ Roasting/smelting step } \\
\hline NOC & 0.00 & 5.3 & 94.7 & 2.63 & 97.37 & 1.26 & 98.74 \\
\hline DC & 22.09 & 1.2 & 98.8 & 1.22 & 98.78 & 0.03 & 99.97 \\
\hline FGS & 22.09 & 0.2 & 99.8 & 0.00 & 99.81 & 0.19 & 99.81 \\
\hline $\mathrm{DC}+\mathrm{FGS}$ & 30.00 & 0.2 & 99.8 & 0.19 & 99.81 & 0.19 & 99.81 \\
\hline $\mathrm{DC}+\mathrm{FGS}+\mathrm{ESD}+\mathrm{SCSA}$ & 95.00 & 13.9 & 86.1 & 70.1 & 29.9 & 13.0 & 87.0 \\
\hline $\mathrm{DC}+\mathrm{FGS}+\mathrm{ESD}+\mathrm{DCDA}$ & 99.33 & 13.9 & 86.1 & 70.1 & 29.9 & 13.0 & 87.0 \\
\hline $\mathrm{DC}+\mathrm{FGS}+\mathrm{ESD}+\mathrm{DCDA}+\mathrm{DFGD}$ & 99.75 & 11.3 & 88.7 & 37.0 & 63.0 & 5.0 & 95.0 \\
\hline $\mathrm{DC}+\mathrm{FGS}+\mathrm{ESD}+\mathrm{DCDA}+\mathrm{WFGD}$ & 99.75 & 3.4 & 96.6 & 37.0 & 63.0 & 5.0 & 95.0 \\
\hline $\mathrm{DC}+\mathrm{FGS}+\mathrm{ESD}+\mathrm{SMR}+\mathrm{DCDA}$ & 99.33 & 13.9 & 86.1 & 70.1 & 29.9 & 13.0 & 87.0 \\
\hline \multicolumn{8}{|l|}{ Extraction step } \\
\hline NOC & 0.00 & 5.3 & 94.7 & 2.63 & 97.37 & 1.26 & 98.74 \\
\hline $\mathrm{WHB}+\mathrm{ESP} / \mathrm{FF}$ & 22.09 & 0.0 & 0.0 & 14.9 & 86.1 & 14.1 & 86.9 \\
\hline \multicolumn{8}{|l|}{ Reclaiming/refining step } \\
\hline $\mathrm{NOC}$ & 0.00 & 5.3 & 94.7 & 2.63 & 97.37 & 1.26 & 98.74 \\
\hline $\mathrm{WHB}+\mathrm{ESP} / \mathrm{FF}$ & 22.09 & 0.3 & 99.7 & 14.9 & 86.1 & 7.0 & 93.0 \\
\hline $\mathrm{WHB}+\mathrm{ESP} / \mathrm{FF}+\mathrm{FGD}$ & 97.90 & 5.0 & 95.0 & 4.9 & 95.1 & 0.9 & 99.1 \\
\hline
\end{tabular}

Note: NOC - none of control; DC - dust collector; FGS - flue gas scrubber; ESD -

electrostatic demister; SMR - specific mercury removal tower; DCDA - double contact and double absorption tower; DFGD - dry flue gas desulfurization tower; WFGD - wet flue gas desulfurization tower; WHB - waste heat boilers; ESP - electrostatic precipitator; FGD - flue gas desulfurization tower. 


\section{S3. Formation of SOX in the furnace}

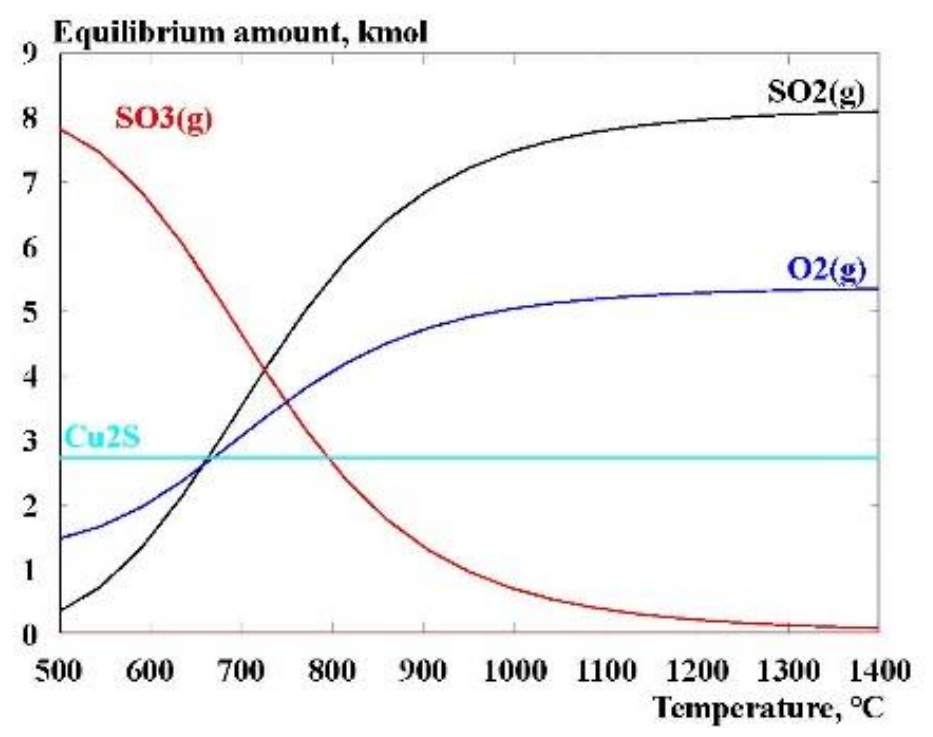

Figure S2. Formation of SOX in the furnace at reaction equilibrium state in smelter C. 